\title{
Translating Performance: Desire, Intention and Interpretation in Photographic Documents
}

\author{
Helen Newall, Amy Skinner and Allan Taylor
}

Since the 1990s, weight has been given to the reception of the photographic document and the phenomenological experience of observing such an artefact. ${ }^{1}$ Debates about documenting performance have focused on the document's relationship with its respective audiences, and this mode of investigation has positioned the camera and its operator as an accidental or passive witness receiving a performance happening in front of the lens, and where the photographs produced are positioned as a by-product, supplement or representation of the live moment. ${ }^{2}$

Furthermore, the advent of digital photography and the subsequent decline of analog photography has not only altered image-making processes but is changing the way in which images are received. This chapter therefore interrogates the notion that digital is any less reliable than analog photography by discussing interventions in the phases of capture common to both formats; and by situating these interventions as a palimpsestic feature of photo-documentation. These interventions comprise: the subject in front of the camera; the photographer and the camera itself; and the spectator or academic scholar examining, receiving and making meaning out of visual information. The construction and editing of photography by subject and photographer is considered in terms of intention; and the authors also consider the notion that if the act of photography, and its resulting images, have 
their own visual language, then the creation of a performance image can be considered a form of transcription ${ }^{3}$ which has implications for how images are read (and particularly the lack of universality in readings).

\section{How the subject intervenes}

Multiple photographs of a single event taken by different photographers reveal, as Ritchin notes, that 'reality itself has no single truth' (2009: 147). Such postmodern constructivist doubt might appear useful when photographs are commonly perceived as 'perfect analogons' of their subjects (Snyder and Allen, in Lister 1995: 10): visual literacy theories have long reinforced the necessity of perceptual scepticism (Sturken and Cartwright 2003). Nevertheless, in an era characterized by what Mitchell has termed 'an ontological aneurism - a blowout in the barrier separating visual fact and fantasy' (1994: 188), any doubt in the integrity of photographic images seems to have shifted from the photograph per se to the digital photograph, most especially since the advent of Photoshop. Analog photography appears less manipulated - the process of sunlight reflecting from an object, travelling through a lens, reacting with chemicals in film emulsion seems uninterruptable and incorruptible, and the darkroom a mysterious space in which a latent image was revealed. The computer, however, is an interface in which a digital image is coded and constructed. Photography's new 'truth myth' would have us believe that analog technology was thus more reliable than its digital successor. But as Lister notes, '[c]ultural theorists have become (often disingenuously) preoccupied with the "loss of the real"' (1995: 1), when there was, perhaps, no 'real' to lose.

That this dichotomy between analog and digital technologies is false (in Lister's phrase, 1995: 8), is evident only one year after 
the official French announcement of photography's invention: in 1840, Hippolyte Bayard staged three photographs of the same subject, all called, Le Noyé or Self Portrait as a Drowned Man, and which quite possibly constitute the first photographic hoax. Complete with caption claiming the subject to be a suicide, they are Bayard's protest at not being officially recognized as one of photography's principal inventors (those named in the French announcement being Daguerre and Niépce).

Bayard's many self-portraits indicate an awareness of the selfconstructive possibilities of photography (and perhaps its defiance of time and death). But besides being the first hoax, Le Noyé with the capture of 'the self-styled body perform[ing] for the camera' (Levin 2015: 328) also offers an early - if not the earliest - example of photographic performativity, for, as Batchen remarks, the composition is calculated, with objects placed carefully within the frame and the subject's pose parodying David's painting Death of Marat (1999: 157-73). Batchen also notes that to replicate the painting via an inverting direct positive print process, Bayard would have had to consciously reverse his pose (166). Le Noyé thus documents the photograph's subject tampering with 'reality' in a calculated performance where the emphasis looks to the aesthetic effect of the end result rather than back at the documentary authenticity of the originating scene.

Le Noyé brings to mind Goffman's description of the presentation of self, where individuals request 'observers to take seriously the impression that is fostered before them. They are asked to believe that ... matters are what they appear to be' (Goffman 1990: 28), and sometimes audiences are aware of the contract, and sometimes they are not. At face value, Bayard's photograph shouts his anger: 'You have metaphorically killed me!' A deeper reading, however, is invited by the circumstances leading to its creation, as Sapir comments: 
This historical context suggests that the self-portrait can be read as a crossroads at which issues of recognition, authorship, display, visibility, invisibility, truth and illusion meet and play off of one another ... As such it can be seen as an early critique of the dominant ocularcentric scopic regime of post-Enlightenment modernity, which assumed a transparent relationship between photography and truth.

Sapir 2003: 619-20

Le Noyé is thus an example of the subject's own complicity with the analog camera to affect a viewer's perception, and make a point: ultimately, photographs are taken, and performances made, 'for the benefit of other people' in Goffman's phrase, and photographs facilitate their seeing such performances from a spatial and temporal distance.

Bayard was deploying what Goffman terms a subject's 'cynical' ability to perform, where a player is 'not taken in at all by his own routine' (ibid.). What of subjects who are not consciously performing? Barthes writes of being photographed: ‘ . . once I feel myself observed by the lens, everything changes' (2000: 10). So, despite a subject's best intentions, the camera can incite a 'performance', and there might be at play an observer effect where the presence of the camera is disruptive and influential. Like an auto-ethnographer, in the act of documentation the camera, whether analog or digital, can become implicated in the process.

Bayard's practice anticipates Baudelaire's scepticism in 1859 when he denounced those credulous enough to believe in photography's 'guarantee of exactitude' (Kriebel 2007: 7), a reproach which itself foreshadows recent criticism of digital pixels being imperceptibly intercepted. Le Noyé thus offers what Barthes and Kuhn deem to be information, but it does not document a drowning, but a performance of a drowning, and certainly the rage of a slighted man. In this it demonstrates photography's facility to be conceptual, and thus more complex than the mirror held up to nature that at its inception some were lauding it to be: the camera documents, but not always what, as 
photographers, as subjects, we assume it is documenting, and regardless of camera, darkroom or computer intervention, the intention of both subject and photographer involved in each image, and which we now discuss, is paramount.

Bayard's performance to camera was never intended to be seen live: it was staged wholly for the photograph. So what of the documenting of performances where the photography parallels the live event? When Amelia Jones offers her idea of 'presence-in-absentia' (1997), she supposes the intention in documentation is to provide an indexical access point to the past in order for a spectator in the present to be taken back to that moment, and she is unapologetic about not having experienced directly the performances she goes on to discuss: 'while the experience of viewing a photograph and reading a text is clearly different from that of sitting in a small room watching an artist perform, neither has a privileged relationship to the historical "truth" of the performance' (Jones 2009: 11). The idea parallels Auslander, who in elucidating the performativity of performance documents, motions that these documents are created during the event to be seen as performances in their own right. Citing Gina Pane and Chris Burden, he states that, 'the events were staged to be documented at least as much as to be seen by an audience' (Auslander 2006: 3). Furthermore, he adds: 'The act of documenting an event as a performance is what constitutes it as such' (2006: 6). Auslander infers, but doesn't fully explore the methods behind, the concept that there is an intention on the part of the artist and the photographer to create a performance that is received in a different temporal space. The suggestion is that before the photograph is even created, the act of documenting anticipates how future spectators will reconstruct or retrieve the information that lies in the visual document at a future time, which poses processual questions to those documenting about how such documents might be constructed. 
A conflict also exists between the prominence of the performer in the analysis of such documents and the relative anonymity of the photographer who has a complicit part to play in this act of performance/document. In Auslander's example of Yves Klein's Leap Into The Void, Klein was undoubtedly the artistic director of the final composition. But this image is in fact a photomontage and thus dependent on the darkroom skills of its photographers. As with Bayard's image, it is also a picture of something which did not occur: the documentation of the creation of this artwork might actually be said to consist of both this and the second image, seen less often, of Klein's friends holding the tarpaulin beneath him as he leaps. And yet, perhaps the finished image is a document, but of a leap from the window Klein claimed he made, without a tarpaulin, earlier that year. There is no proof he made this first leap: it is undocumented. And what of the role of Harry Shunk and Janos Kender, the Hungarian photographers who took the images for him? There are clearly implications in collaboration and impact in the photographer-artist relationship.

Likewise, whether or not the artist is aware of being documented, more academic awareness of intention as a part of the process of documentation is required, for even the act of clicking the shutter on a camera has intention. There are then two questions: why was this particular photograph created, and what did its photographer intend?

But we must also consider selection and what is intended here: who decided which shots represent the performance work? A photographer might select with aesthetic compositional considerations of what makes a good performance photograph; whereas a director, or choreographer, might choose those images which record what they consider key performance points. A performer, however, might select for a portfolio to further a career. In such selections of shots, human agency foregrounds some images over others, thereby erasing an 
unselected subset from the archive memory. Photographs constituting documents might be supplanted by an entirely different intention behind their selection leading to an incongruence in the reading of such a document.

Grant (2002) discusses such issues in her paper 'Private Performances', in which she uses Mendieta and Abramovic to illustrate the heavy editorial control some artists employ to ensure the art represented is as they intended. Such editing strategies have been exposed via photographers such as Hannah Starkey and Hellen van Meene, 'in whose work the authenticity and authority of the photograph has been radically eroded and problematized' (Grant 2002: 34). She goes on:

Through this newer generation of photographic practitioners, the editing process present in the performance documentations of Abramovic, Pane and Mendieta is revealed as a strategic, sophisticated and often overlooked tactic, staged in order to maintain the complexity of these images and descriptions.

Such elements of editing and exhibition clearly embedded into documentation constitute what intentists refer to as 'Palimpsestism" ${ }^{4}$ the trail of the creative journey leading from intention to the finished vehicle, where the resolved work is clearly distinguishable from the work in progress. Nemiroff discusses this as a form of layering:

The textual elements [of the photograph], whether visual or verbal, are most often doubled so that one text is read through another or in juxtaposition to another. Thus, material borrowed from or patterned on popular culture sources is given a new, or supplementary, meaning in the context of their art.

Nemiroff 2005: 47

Thus the photograph becomes several texts written on top of one another until they appear to the audience as one translucent image 
with many different things to read and multiple entry points to interpretation. The individual selects the relevant entry point, reads the most pertinent texts and assimilates them accordingly to produce their meaning. A photograph can thus be many narratives, but artist and/or photographer intention, constructed via context, manifesto, or complementary writing, is a significant and authoritative voice in the weave.

\section{How the photographer and the camera intervene}

In repositioning the photographer as being as important as the artist or work being documented, the debate should perhaps draw on the school of intentism as defined by Pelosi:

[Intentists] believe that their artwork is able to convey their artistic intention to their intended audience ... As a movement it both recognizes and celebrates the relationship between an artist's creation and its creator.

Pelosi 2009: 3

That is to say that though we learn from interpretation and reception of such documents, if the author of these documents is put back into the picture, then an awareness of the impact that inception, creation and process can have becomes possible. When the knowledge that a performance has the potential to be documented exists, there is then an intentional editing and presentation process before the document is exhibited to its wider audience.

If the relationship between artist and photographer is complex, then the relationship between the photographer and camera is equally so, for the camera has sight of the performance it documents; it is the site where the performance is photographically constructed and collated; and ultimately, in the resulting images, it cites the 
performances of others. But it is, as Flusser writes, an apparatus 'programmed' by functions decided by its inventors at spatial and temporal distances. It is also a complicated apparatus around which is often a cult of gadgetry: 'every photograph', Flusser writes, 'is a realization of one of the possibilities contained within the program of the camera' (2000: 26), and '[e]very single photograph is the result, at one and the same time, of co-operation and of conflict between camera and photographer' (2000: 47). In manual mode, the photographer makes decisions about depth of field, shutter speed and aperture. In auto mode, the camera is making these pre-programmed decisions: the jpeg is the result of algorithms processing a photograph on your behalf: 'straight out of camera' is a false concept. Those decoding photographs must therefore ask:

'How far have photographers succeeded in subordinating the camera's programs to their own intentions?' And, vice versa: 'How far has the camera succeeded in redirecting the photographer's intentions back to the interests of the camera program ...?'

Flusser 2000: 47

Ultimately, photographers can only capture what their cameras can photograph, and state of the art technology can still fail. As Jess Allen notes: 'no matter how sophisticated the documentation strategies that digital technology currently offers, they still privilege that which can be documented over the less tangible information gained from live interaction' (Allen 2010: 67). The first war images were, for example, static landscapes rather than action shots, because cameras at that time required lengthy exposures. The first theatre images were similarly defined by the limits of contemporaneous camera technology. Taken not on theatre stages amid the action of rehearsal or performance, these were staged in photographic studios before painted backcloths appropriate to the play, lit by skylights, using the photographer's own props and furniture. The performers posed 
statically as characters, and were sometimes costumed in clothing borrowed from the studio (Shields: online). But these were publicity photographs taken to sell tickets rather than document performance.

By the early 1900s, the desire for such photographs had increased while exposure times had reduced. Nevertheless, images of Pavlova by Herman Mishkin still involved wires and contraptions to stabilize her while she held poses en pointe long enough for Mishkin to expose the glass plates (Mitchell 1999: 70). However, as Senelick notes: 'a given pose is caught but remains segregated from what went before or came after. The image segments a discrete emotional climax, distilling and fixing it in the process' (Senelick 1997: 256). This is true of any photograph capturing either a posed moment, or one captured in the flow of a scene. They are, in Senelick's terminology, crystallized out of context (256). Thus, as photographer Jack Mitchell notes, these images 'reveal much about each dancer - except how they appeared in motion' (1999: 70).

In the end, these early images constitute performances of performance, and in Postlewait's terms they are: 'unreliable testimony because both their final cause or aim (publicity shot) and their formal cause (aesthetic principles of portrait) subvert their documentary potential' (2009: 245). But for the most part they are the only extant photo-documentation: any faulty information comes from faulty positioning of images which were primarily about selling celebrity and tickets, and in this sense they are documentation of how early the Faustian pact between the famous and the camera began. ${ }^{5}$

\section{The intervention of the spectator}

We now turn our gaze to the spectator, the academic in the archive, viewing images. For such academics, the advent of digital photography and the increasing online presence of performance archives have, in 
combination, led to an historiographical cognitive schism in the study of performance history concerning: 'not only the methods that define and guide the practice of historical study and writing but also the self-reflexive mind-set that leads us to investigate the processes and aims of historical understanding' (Postlewait 2009: 2). This collision between objective methodologies and their application by subjective mind-sets is sited within the academic. But the academic's scope concerns equally fragmented objects of enquiry: for in such an era as ours of unprecedented access to photographic material, performance photographs are both historiographically valuable and problematic documents, and, as Grant has already stated, academics often find themselves dealing not with original performances, but with their traces. Sallenave observes, 'our memories of theatre are frequently memories of photographs of theatre' (in McAuley 2008: 9).

The shift from analog to digital photography marks a significant watershed in performance historiography. The development of a new mode of photographic production brings a concurrent change in photographic currency: photographs are differently perceived because they are differently produced.

This is particularly apparent in the relationship between photography and temporality. For Barthes, writing before the advent of digital photography, temporality is key in the negotiation of the relationship between the referent and the reception of the photographic image. The photograph is 'a new form of hallucination: false on the level of perception, true on the level of time: a temporal hallucination, so to speak, a modest, shared hallucination (on the one hand "it is not there," on the other "but it has indeed been")' (Barthes 1981: 115). Barthes's 'temporal hallucination' in fact embodies the multiple temporalities found in the photographic image, a collapse of the moments of production and reception, as he explores in his observations of Salzmann's photographs of Bethlehem: 'Nothing but stony ground, olive trees; but three tenses dizzy my consciousness: my 
present, the time of Jesus, and that of the photographer, all under this instance of "reality"' (1980: 97).

The photographic image, outside of the mechanisms of production involving film emulsion or pixels, is suffused by temporal structures. Engagement with these structures is clearly inflected by the context in which the photograph is viewed: Barthes's Camera Lucida revolves around the evocative power of a shared time and the tension embedded in the distance between image production and reception. In collapsing this distance, the digital era has bought about shifts in temporal understanding towards new models of 'sharing', temporalities of 'immediacy', 'acceleration' and instantaneity (The Experience of Time in Modern Culture, University of Warwick). ${ }^{6}$ If, as Bluedorn notes, 'time is used to generate meaning' (2002: 42), then changes in contemporary understandings of time alter potential engagements with the temporality of the photograph, and particularly of the historical photograph.

The close relationship between temporality and culture (as advocated, for example, by Ricoeur 1984), suggests that different temporal structures can complicate the understanding of a photograph passing from one temporal context to another. Familiarity with the overt formal photographic structures (how the image appears to look, and its constructed transparency, the so-called 'truth myth') can disguise this potentially significant moment of alienation. As such, the shift from analog image-making and Barthes's sharing, towards digital photography and the instantaneity of digital culture, can be seen as a moment of schism: for how photographic practice functioned at the moment of the image's creation is not the same as how it functions in the contemporary moment of reception, and, as the digital revolution accelerates, photography's 'truth myth' shifts with it, acquiring hitherto unanticipated nuances which we will now discuss. 


\section{Photography and its intentional gaps}

Mitchell says of the relationship between language and the image that 'pictures want equal rights with language, not to be turned into language ... To be seen as complex individuals occupying multiple subject positions and identities' (2005: 38). If it is then considered that performance, and its documentation via photography, have an equivalent status to language, then Derrida's idea of différance $(1988)^{7}$ can be used to interrogate why it is employed to document performance. Derrida describes the relationship between speech and thought as much more closely related: speech happens as though we are hearing our own thoughts again in a more simultaneous or instantaneous matter. Writing, however, happens as a much more considered process and therefore has a much further distance from the original thought. Nonetheless, it is constructed in the knowledge that when it is read, it will be enacted once again in the minds of those who read it.

In the same way, thought and performance acts have a much more simultaneous relationship, but it is then 'written' by photography, which isolates it from the event and distances it to a point ready to be received - ready to be performed - at a latter moment. In both cases, what occurs is a 'deferred performance': a way of transcribing the act or utterance so that when it is seen again, it can be enacted once more. This is the intentional gap, or 'double now' that Phelan notes echoing Barthes's multiple temporalities (2010).

If looked at as intentional practice, it is reasonable to assume that this gap is anticipated by its creator and used purposefully. The 'performative power' of the photograph relies on these gaps and is discussed by Baetans (2009) as lacunae or 'missing text.' Explaining that the photograph 'shows' rather than 'tells', this mechanism of 'showing' allows an audience to fill in the blanks and come up with individual connections to, and performance of, the still image. He writes: 
What needs to be stressed is the position that 'lacunae' (vacancies, blanks, uncertainties) have acquired within the narrative impulse, becoming a foundation of narrative reading. Put simply, the more any one medium [for example, film] attempts to explain, justify, unfold or conclude its own narrative, the more its narrative power diminishes.

Baetans 2009: 146

When set into juxtaposition with photography, film dictates to the viewer exactly how the narrative arc plays out. In the still image, ranges of responses are possible and - in fact - invited. The artist makes these gaps by layering several texts: the performance act, the setting and the pose. This is, again, is palimpsestism - a trail that academics in the archive need to unravel. Of course, this brings into question the transcription process: if what is written by photography is not consistently read in the same way, how do we resolve this transcriptioninterpretation relationship? In response, it is perhaps useful to suggest a new model for photographic documentation, substituting ideas of performance documentation, with those of performance translation. The researcher can turn the photographic document into a process of documentation through conscious engagement with the image. In the archive, for example, this engagement can include metadata (such as company records, or the name of the photographer). The model of translation contributes to this process and, in some instances, actively creates the meta-context for the engagement with performance photographs, allowing the researcher to turn a photographic document into a process of documentation.

\section{The photograph as object of translation}

More than a simple re-naming, this new terminology implies a different conceptual relationship between the photograph and the 
researcher: one of active interpretation and reading which brings the moment of photographic creation into the moment of reception. Translation principles, in fact, underwrite two aspects of photographic historiography in performance: firstly, the photograph itself functions as a translation of the performance event; secondly, the researcher reading the photograph also engages in his or her own process of performance translation. It is this processual act that allows the photograph to perform the function of documentation.

For the performance scholar, the attraction of the photograph is not necessarily its preservation of the performance moment, but what Reason calls its 'transformational truth' (2008: 7), the re-working of the event into a different temporal and spatial frame: photographs of performances are a meeting point of the different visual languages associated with different creative media. By inflecting Reason's use of 'transformation' to the very specific transformative process of translation, salient qualities of the translated text can be applied to photographic documentation, in particular, the consciousness of process and resulting multiplicity embodied in the translated object.

The process of linguistic translation holds in tension the relationship between a text's producing and receiving cultures. ${ }^{8}$ The extent to which the producing culture is linguistically embedded in the translated text is addressed by David Bellos:

If a detective novel set in Paris makes its characters speak and think in entirely fluent English - even while they plod along the Boulevard Saint-Germain, drink Pernod and scoff a jarret de porc aux lentilles then something must be wrong. What's the bonus in having a French detective novel for bedtime reading unless there's something French about it?

2011: 41

In other words, the translated text mediates between two languages and cultures. As Bellos notes, the pleasure in the French detective story is, in part, its 'Frenchness' - and the language of the translation 
mediates that Frenchness to the receiving culture. It is the tension between the familiar and the strange, the sense of located and embedded otherness that allows the translated text to function as a (conscious act of) translation. There is something provocative in this foreignness that shifts the reader's perception.

This question of a conscious negotiation of two contexts can equally be applied to the photograph, which becomes an object of mediation. The photograph collapses its own visual language with that of the performance. As with the translated text, it is only when both of these (visual) languages are acknowledged that the photograph 'makes sense', and becomes useful in the process of performance documentation. The translator makes a series of choices that construct the reception of the text (such as those referenced by Bellos); similar choices are apparent in photographic practice. The photographer frames, excludes and directs the eye: like the translator, the photographer's role is primarily that of mediation, transforming the language of performance into the language of photography.

\section{Photographic Reception as Translation}

In addition to its mediation between different media - the 'languages' of performance and photography - the photographic object is also the product of a specific cultural and visual moment. How a photograph is understood is a balance of familiarity and difference: the photograph is the connection point between here and now, and then and there (Barthes's multiple photographic tenses). If photography asks for equivalency to language, then it responds just as much to context as its counterpart, in the same way that an art form like painting does. As such, the photograph is not transparent: not only in the sense that it is not a transparent representation of 'truth', but in the sense that it is not temporally or spatially universal. Where photographic practice can be attributed to a specific (and often well-known) photographic 
artist, the need to acknowledge a wider aesthetic influence on the construction of the image is readily apparent. However, much historical photography is unattributable, distancing the image from the cultural-aesthetic conditions of its production. As such, it is not only necessary to develop an understanding of the intentions of an individual photographer, but also of the context in which that photographer's practice was developed. Photographs are not just received: they are read.

The visual culture from which the photograph emerges is an essential frame of reference for understanding the images. This extends beyond observations around the intended use of the photograph - for advertising or documentation purposes, for example - to the specific qualities of certain photographic moments. These moments are born out of both developments in visual culture and in advancing photographic technologies: in the early twentieth century, photo secession increased photographic pictorialism, early mass-use cameras flattened perspective or caused a halation effect, surrounding objects with a halo of light (both features put to specific artistic use by Picasso), 1920s Russian experiments in photomontage suggested photography as an interrogative and fragmented formal process. ${ }^{9}$ Each moment inflects the construction of the photographic image - and images of theatre and performance are not immune to this influence. To use a photograph for the purposes of historiography requires the researcher to consider not only the broad differences between the photograph and performance space, but also the specific inflections of photographic practice at different historical moments. In other words, a photographic language must be identified and contextualized. The photograph must be translated.

\section{Motion, flux and the need for consciousness}

Barthes's understanding of photography as a temporal phenomenon highlights the relationship between movement and photographic 
practice. The question of capturing movement is at the heart of photography, and the suspension of movement in the photograph creates images that are static, but not still, radiating motion from the captured instant, what Hart Crane calls 'the moment made eternal' (in Sontag 1979: 65): photographs are objects in motion. Nowhere is this more obvious than in their usage by performance scholars. Beyond the photograph's engagement with motion associated with the performance event, Barthes's articulation of the image in 'three tenses' implies a potential sense of flux embedded in photographic reception. The image is constantly changing; re-read in light of developing understandings of its context of creation and its context of reception. This sounds obvious, but it implies that images require translation both in and out of contexts. The journey from photomaking to photo-using is a vital one in understanding photographic reception, and when photographs are used as documentation, they become a bridge between the producing and receiving cultures. This raises a fundamental question around photographic translation: what is being translated? Is the photograph a translation of a visual experience of a performance, and thus rooted in an image, or is it the embodiment of a theatrical idea and process? If it is the latter, this implies a shift in emphasis from image to idea, changing our relationship with the object from what it represents to what it contains.

This shift allows for an engagement with photographic documentation that takes into account not just the content of the documented performance, but also the purpose of the act of performance analysis within the context of the receiving culture. The significance of the dual cultures of the translated text are perhaps most apparent in the debate on the purpose of translation. Sirkku Aaltonen frames the two reflexive models of translation as the window and the mirror (2000: 1). Both analogies are problematic, and serve primarily to highlight the egocentric function of much translation 
practice, framed as a reflection on the self of the receiving culture far more than on the other of the producing culture.

The window/mirror analogies are reflections on the purpose of linguistic translation that can equally be applied to the historiographic use of photography as a process of documentation: to what extent are observations made based on photographs of historical practice shaped by the concerns of contemporary performance, and, to what extent does this inflect the analysis of the historical with a contemporary agenda?

As such, the application of translation theory to photography allows us to question the documentary purposes of these 'translated objects' and the narratives that they prompt us to create. As developments in photographic technologies reframe cultural understandings of the photograph, images increasingly become accessible through online archiving, and shifts in temporal-cultural models alter our relationship with the images that the camera can produce, a consciousness in the use of the process of documentation through photography becomes more and more essential. Above all, translation as a model allows us to see how the historiographic potential of the performance photograph must be handled with increasing care.

Documents are thus as unfixed as the status of the photograph itself, and depend on questions an interlocutor asks of it in an act of translation: the implications for the performance scholar in dealing with palimpsestic texts and photographic transcriptions of performance. 
